\title{
Des enjeux importants pour les patients - aussi après le 1er janvier 2012
}

\author{
Il est probable que l'entrée en vigueur de la nouvelle LAMal causera des problèmes \\ considérables en lien avec le financement d'hospitalisations hors canton, surtout \\ dans la phase initiale. Une fausse idée répandue dans la population et parmi nos \\ confrères veut que les frontières cantonales soient totalement supprimées avec la \\ nouvelle loi, ce qui risque de conduire à des hospitalisations qui ne remplissent pas \\ les conditions nécessaires à la prise en charge des coûts par l'assurance obligatoire \\ des soins et par les cantons. L'article suivant donne des recommandations* pour évi- \\ ter ce genre de problèmes.
}

\section{Thomas Plattner ${ }^{\text {, }}$ \\ Magdalena Wicki Martin ${ }^{b}$, Robert Gmürc, \\ Chung-Yol Leed \\ a Dr méd., Spécialiste en médecine légale, Médecin cantonal adjoint, Fribourg \\ b Collaboratrice scientifique, Service de santé publique, Fribourg \\ c Conseiller juridique, Service de santé publique, Fribourg \\ d Dr méd, Spécialiste en médecine interne, Médecin cantonal, Fribourg}

\section{* Voir en particulier le paragraphe «Conséquences et recommandations aux} médecins» à la fin de l'article.

Correspondance: Dr Thomas Plattner Service du médecin cantonal Chemin des Pensionnats 1 CH-1700 Fribourg Tél. 0263057980

thomas.plattner@fr.ch

\section{Introduction}

Parallèlement à l'introduction d'un nouveau système tarifaire, la révision actuelle de la LAMal poursuit, en lien avec le financement hospitalier, le but d'assurer le libre choix du prestataire pour tous les résidents et résidentes suisses [2]. Pour cette raison, la différenciation entre «hôpitaux publiques» et «cliniques privées» a été abandonnée et l'obligation de la prise en charge des coûts par l'assurance de base et les cantons a été étendue à tous les hôpitaux figurant sur une liste cantonale.

Le fait que les coûts couverts par les cantons ne se limitent, dans certaines situations, qu'au tarif intracantonal de référence n'est, de notre expérience, pas suffisamment connu du corps médical. Cette disposition peut pourtant signifier qu'une éventuelle différence des coûts soit facturée au patient, ou, subsidiairement, à son assurance complémentaire. Ce qui nous semble donc problématique, c'est que tant parmi les assurés que parmi les professionnels de la santé, l'avis semble de prévaloir que toutes les frontières cantonales en lien avec le financement hospitalier soient supprimées et que chaque patient puisse se faire traiter dans toute la Suisse, sans assurances complémentaires et sans garantie de paiement par le canton de résidence.

Par conséquent, il sera désormais d'autant plus important que les médecins - en cabinet et en milieu hospitalier - soient bien informés des conditions d'une participation des cantons aux coûts d'un traitement stationnaire, de manière à ce qu'ils puissent à leur tour informer leurs patients correctement. Ciaprès, nous aimerions expliquer les nouvelles «règles du jeux» - telles que nous les interprétons actuellement - et donner quelques indications pratiques aux médecins.

\section{Bases légales et procédures administratives} Dès 2012, conformément à l'art. 39 de la LAMal, tous les hôpitaux figurant sur la liste d'un canton (hôpitaux répertoriés) ou qui ont un contrat avec un assureur (hôpitaux conventionnés) seront autorisés à pratiquer à charge de l'assurance maladie de base et cela indépendamment de leur statut légal, qu'il s'agisse d'un hôpital privé ou d'un hôpital public ou subventionné par les pouvoirs publics.

Désormais, et dans le cadre de leur planification hospitalière, les cantons tiendront une liste de tous les prestataires stationnaires qu'ils estiment nécessaires à la couverture médicale de leurs résidents. Sur le principe, chaque canton est libre de choisir les prestataires qu'il veut mettre sur cette liste. Dans les faits, les cliniques et hôpitaux extra-cantonaux, voire certains départements ou même certaines prestations spécifiques ne seront admis sur cette liste que s'ils correspondent à un besoin non couvert par l'offre de prestations du canton.

Pour les traitements stationnaires extra-cantonaux et à condition de figurer sur sa liste accréditée, le canton couvrira au minimum $55 \%$ des coûts, et cela indépendamment du canton d'implantation de l'hôpital en question. Le coût résiduel sera couvert par l'assurance de base. Dès 2012, les patients pourront choisir librement le prestataire parmi les hôpitaux figurant sur la liste du canton de résidence (hôpitaux répertoriés) sans passer par une procédure de garantie de paiement.

En revanche, pour assurer une couverture complète de la part cantonale lors de traitements dans un hôpital figurant uniquement sur la liste du canton d'implantation ou d'un hôpital qui se trouve sur la liste d'un canton tiers ou encore d'un hôpital conven- 
Tableau 1

Modalités de financement.

\begin{tabular}{|c|c|c|c|c|}
\hline \multirow{2}{*}{$\begin{array}{l}\text { Art. LAM } \\
\text { (nouveau) }\end{array}$} & \multirow[t]{2}{*}{ Hôpital } & \multicolumn{2}{|c|}{ Garantie de paiement par le canton } & \multirow[t]{2}{*}{ Répartition des coûts } \\
\hline & & Urgence* & Traitement électif & \\
\hline $\begin{array}{l}41.1 \text { et } \\
\text { suivants }\end{array}$ & $\begin{array}{l}\text { Hôpital sur la liste du } \\
\text { canton de résidence }\end{array}$ & Pas nécessaire & Pas nécessaire & $\begin{array}{l}\text { Assurance obligatoire de soins + } \\
\text { canton de résidence }\end{array}$ \\
\hline \multirow[t]{2}{*}{$\begin{array}{l}41.1 \text {. et } \\
\text { suivants } \\
41.3\end{array}$} & $\begin{array}{l}\text { Hôpital sur la liste du } \\
\text { canton d'implantation }\end{array}$ & $\begin{array}{l}\text { Dépend du } \\
\text { canton, mais } \\
\text { recommandée } \\
\text { de manière } \\
\text { générale }\end{array}$ & Nécessaire & $\begin{array}{l}\text { Urgence/garantie de paiement octroyée: } \\
\text { Assurance obligatoire de soins + } \\
\text { canton de résidence }\end{array}$ \\
\hline & & & & $\begin{array}{l}\text { Pas d'urgence/garantie de paiement refusée: } \\
\text { Assurance obligatoire de soins + } \\
\text { canton de résidence } \\
\text { (au maximum jusqu'au tarif référence du } \\
\text { canton de résidence) + patient/assurance } \\
\text { complémentaire }\end{array}$ \\
\hline \multirow[t]{2}{*}{49 a al. 3} & \multirow[t]{2}{*}{$\begin{array}{l}\text { Hôpital sur la liste } \\
\text { d'un canton tiers** }^{*}\end{array}$} & \multirow[t]{2}{*}{ Nécessaire } & \multirow[t]{2}{*}{$\begin{array}{l}\text { Pas de participation } \\
\text { financière par le canton }\end{array}$} & $\begin{array}{l}\text { Urgence/garantie de paiement octroyée: } \\
\text { Assurance obligatoire de soins + } \\
\text { canton de résidence }\end{array}$ \\
\hline & & & & $\begin{array}{l}\text { Pas d'urgence/garantie de paiement refusée: } \\
\text { Assurance obligatoire de soins + } \\
\text { patient/assurance complémentaire }\end{array}$ \\
\hline \multirow[t]{2}{*}{49 a al. 3} & \multirow[t]{2}{*}{$\begin{array}{l}\text { Hôpital } \\
\text { conventionné } e^{\star \star \star}\end{array}$} & \multirow[t]{2}{*}{ Nécessaire } & \multirow[t]{2}{*}{$\begin{array}{l}\text { Pas de participation } \\
\text { financière par le canton }\end{array}$} & $\begin{array}{l}\text { Urgence/garantie de paiement octroyée: } \\
\text { Assurance obligatoire de soins + } \\
\text { canton de résidence }\end{array}$ \\
\hline & & & & $\begin{array}{l}\text { Pas d'urgence/garantie de paiement refusée: } \\
\text { Assurance obligatoire de soins + } \\
\text { patient/assurance complémentaire }\end{array}$ \\
\hline
\end{tabular}

* Le traitement doit être administré sans tarder et un transfert/une admission dans une clinique de la liste du canton de résidence n'est médicalement pas justifiable/possible.

** Cliniques qui se trouvent ni sur la liste du canton de résidence ni sur la liste du canton d'implantation mais sur la liste d'un autre canton (par ex. cliniques de réhabilitation dont le propriétaire ne se trouve pas sur le sol du canton d'implantation).

*** Cliniques qui ont un contrat avec un ou plusieurs assureurs mais qui ne figurent sur aucune liste cantonale.

tionné (définitions voir tableau 1), une garantie de paiement demeure nécessaire. Pour ces hôpitaux, à défaut d'une garantie de paiement et selon l'art. 41 de la LAMal, le canton de résidence ne paiera que la partie correspondant au tarif de référence intra-cantonal (à l'exception des hôpitaux conventionnés où le canton ne participe pas aux traitements électifs). Il incombera donc au patient lui-même ou à son assurance complémentaire de s'acquitter du montant restant (voir tableau 1). Puisqu'il faut s'attendre, même avec un système tarifaire unique, à de grandes variations de tarifs entre les cantons, d'importantes conséquences financières pour les patients ne peuvent être exclues, notamment dans les cantons pratiquant des tarifs inférieurs à ceux des cantons voisins.

Comme aujourd'hui, la garantie de paiement sera octroyée s'il existe une raison médicale. Sont réputées raisons médicales les urgences et des traitements qui ne sont pas disponibles dans le canton de résidence du patient. Il appartiendra, comme aujourd'hui, à un médecin officiel, en règle générale le médecin cantonal, de décider si ces conditions sont remplies et d'autoriser la participation financière par le canton.
Selon la loi, les urgences sont en théorie exclues de cette nécessite d'une garantie de paiement. Or, le législateur ne définit toujours pas ce qu'il entend sous «urgence». Une interprétation fidèle à une décision du Tribunal administratif fédéral induirait que l'urgence est à considérer que si l'état de la personne qui doit être traitée ne permet pas son transfert dans un hôpital répertorié (figurant sur la liste du canton de résidence).

Ce qui est une urgence et ce qui ne l'est pas est donc une question d'interprétation. Face au risque d'abus de recours à la notion d'urgence pour bénéficier de la participation cantonale au financement d'hospitalisations hors-canton non justifiées, les cantons vont certainement accroître leur vigilance afin de vérifier le caractère d'urgence d'une admission indiquée comme telle et, le cas échéant, refuser la créance. La plupart des cantons vont sans doute insister sur une demande de garantie de paiement même pour les urgences. Concrètement, cela signifie que le patient qui ne dispose pas d'une assurance complémentaire couvrant la prise en charge des traitements dans toute la Suisse devra de préférence se rendre 
dans une clinique figurant sur la liste de son canton de résidence, pour autant que la prestation y soit disponible, même en situation d'urgence, sauf s'il y a nécessité médicale d'un traitement immédiat dans la clinique non répertoriée la plus proche. De même, lors d'un traitement ambulatoire dans un autre canton, il y a lieu d'anticiper la survenue éventuelle de complications nécessitant une hospitalisation. En effet, le patient qui choisit un traitement ambulatoire dans une clinique non-répertoriée et sans nécessité médicale (le traitement aurait pu se faire dans une clinique de la liste de son canton de résidence) ne pourra pas recourir à la totalité de la participation financière par le canton en cas de complications qui nécessiteraient un traitement stationnaire hors canton [3].

Le tableau 1 (page 1586) qui suit tente de vous donner une vue d'ensemble des variantes possibles, et ceci en l'état actuel de nos connaissances; il n'est par conséquent pas exhaustif.

\section{Conséquences et recommandations aux médecins}

Il est probable que l'entrée en vigueur de la nouvelle LAMal causera des problèmes considérables en lien avec le financement d'hospitalisations hors canton, surtout dans la phase initiale. Une fausse idée répandue dans la population et parmi nos confrères veut que les frontières cantonales soient totalement supprimées avec la nouvelle loi, ce qui risque de conduire à des hospitalisations qui ne remplissent pas les conditions nécessaires à la prise en charge des coûts par l'assurance obligatoire des soins et par les cantons.

Pour leur éviter des problèmes, il est demandé aux assurés, qui doivent au final assumer eux-mêmes la responsabilité du choix d'un hôpital et les éventuelles conséquences financières, de maîtriser la notion d'urgence, de connaître l'offre médicale de leur canton de résidence et d'être au fait de la liste cantonale des hôpitaux. De telles exigences ne sont à notre avis pas acceptables à l'égard des patients, d'où l'importance que nous accordons à une bonne information des médecins.
En respectant ces quelques indications, des problèmes devraient en principe pouvoir être évités:

- De manière générale, adressez vos patients à une clinique figurant sur la liste du canton de résidence, à moins que:

- L'admission dans un hôpital hors de la liste du canton de résidence s'avère médicalement nécessaire (urgence ou/et prestation pas disponible dans une clinique sur la liste du canton de résidence, voir liste négative du canton de résidence ou se renseigner auprès de l'hôpital de référence du canton de résidence).

- Le patient dispose d'une assurance complémentaire qui assure le traitement dans toute la Suisse et souhaite l'admission dans une clinique non-répertoriée par le canton de résidence.

- Examinez l'indication d'urgence. Une urgence au sens de la loi se présente uniquement lorsque l'état du patient ne permet pas son (re)transfert dans une clinique figurant sur la liste du canton de résidence.

- Soumettez toujours une demande de garantie de paiement au canton pour les admissions en cliniques ne figurant pas sur la liste du canton de résidence et cela aussi en cas d'urgence et aussi pour des patients qui disposent d'une assurance complémentaire. Pour des interventions électives, cette demande doit être soumise suffisamment tôt, au minimum une semaine avant l'admission. Complétez entièrement le formulaire ad-hoc et de manière lisible. Les demandes lacunaires ou non-lisibles seront renvoyées.

\section{Références}

1 Partie I: Plattner T, Lee CY. Hospitalisations extracantonales - des enjeux importants pour les patients. Bull Méd Suisses. 2009;90(41):1588-9.

2 Au profit d'une meilleure lisibilité, la dénomination au masculin est désormais utilisée.

3 Décision du Tribunal administrative fédéral 14 octobre 2002 (AFTA K 128/01).

4 Décision du Tribunal administrative fédéral 13 avril 2006 (AFTA K 81/05). 